Portland State University

PDXScholar

\title{
Subwavelength Visualization of Light in Thin Film Waveguides with Photoelectrons
}

Joseph P. Fitzgerald

Portland State University, fit@pdx.edu

Robert Campbell Word

Portland State University, wordr@pdx.edu

Rolf Könenkamp

Portland State University, rkoe@pdx.edu

Follow this and additional works at: https://pdxscholar.library.pdx.edu/phy_fac

Part of the Condensed Matter Physics Commons

Let us know how access to this document benefits you.

\section{Citation Details}

Fitzgerald, J. P. S., Word, R. C., \& Könenkamp, R. (2014). Subwavelength visualization of light in thin film waveguides with photoelectrons. Physical Review B, 89(19), 195129.

This Article is brought to you for free and open access. It has been accepted for inclusion in Physics Faculty Publications and Presentations by an authorized administrator of PDXScholar. Please contact us if we can make this document more accessible: pdxscholar@pdx.edu. 


\title{
Subwavelength visualization of light in thin film waveguides with photoelectrons
}

\author{
J. P. S. Fitzgerald, ${ }^{*}$ R. C. Word, and R. Könenkamp \\ Department of Physics, Portland State University, 1719 SW 10th Avenue, Portland, Oregon 97201, USA
}

(Received 8 January 2014; revised manuscript received 5 May 2014; published 21 May 2014)

\begin{abstract}
We report the visualization and quantitative analysis of electromagnetic surface fields at solid surfaces with the potential for $\lambda / 50$ resolution. To illustrate this capability, we investigate patterns in two-photon photoemission images of light-diffracting structures in waveguiding, transparent thin films. The obtained micrographs show interference patterns between incident and guided light with a remarkable sensitivity to subwavelength features. We demonstrate that photoemission rates are directly related to the surface field strengths and develop a subwavelength method to calculate the surface fields from optical properties and surface topology based on the two-dimensional Kirchhoff diffraction integral. Calculated images based on this theoretical approach compare favorably to experimental electron micrographs.
\end{abstract}

DOI: 10.1103/PhysRevB.89.195129

PACS number(s): 68.37.Xy, 42.25.-p, 42.82.Et, 78.67.-n

\section{INTRODUCTION}

In the past decade the optoelectronics field has attracted considerable research interests following new materials developments and experimentation techniques. With the emergence of nanoscale plasmonic and photonic devices there is a large interest for quantitative characterization. Scanning probe techniques, particularly scanning optical microscopies, have largely been used to address these needs. More recently, however, photoemission electron microscopy (PEEM) has been developed towards a level where images with high spatial and temporal resolution can be obtained [1-9]. With the availability of ultrashort high-intensity laser pulses, even the infrared and visible spectral region can be routinely used in PEEM when multiphoton excitations are utilized. This makes PEEM a potentially powerful tool for the study of all types of electromagnetic field excitations in materials, including guided optical modes [6,9], vacuum modes [7], and surface plasmons [1-5,8]. With its excellent spatial resolution now approaching $5 \mathrm{~nm}$ [10], PEEM may advance optical surface studies to scales well beyond standard optical microscopes and beyond currently available super-resolution techniques. Here we present a detailed analysis of light propagation in nonmetallic transparent media observed in two-photon (2P) PEEM at a wavelength of $410 \mathrm{~nm}$.

Conventional low-energy PEEM uses ultraviolet light to induce photoemission from surfaces via single photon excitation processes. In PEEM, this emission pattern is imaged with electrons [11]. Since electrons are used to create the image in PEEM, the fundamental resolution limit is set by the electron optics, as in other electron microscopies, and is unaffected by the diffraction limit of the light used to illuminate the sample [12]. Multiphoton PEEM ( $n$ P-PEEM) extends the wavelength range of incident light to the visible and infrared. Multiple $(n)$ photons are used to overcome the work function $W$ in the electron excitation, i.e., $n \times$ $(h c / \lambda)>W$. Multiphoton excitation is a low-probability, nonlinear process that is sensitive to local variations in the electromagnetic field, allowing phase-resolved visualization of surface electromagnetic fields [5,6].

*fit@pdx.edu
Presently, $n \mathrm{P}$-PEEM is primarily used to visualize surface plasmon polariton fields $[4,5,8]$. Here we show that $n \mathrm{P}-\mathrm{PEEM}$ is also suitable for visualizing the surface photonic fields of two-dimensional structures in transparent waveguides, showing remarkable sensitivity to subwavelength features. Building on an earlier analysis of a single slit in a dielectric slab waveguide [6], we present a method to calculate the diffraction patterns observed in PEEM. We establish the relationship between electromagnetic field intensity and multiphoton photoelectron emission yield and develop a procedure for calculating surface fields from scalar wave diffraction theory.

In our analysis, we first determine the wave numbers of guided modes that can be expected in a dielectric thin film, then use Fraunhofer diffraction to determine the relative amplitude of modes excited at the periphery of the film where the in-coupling occurs. The evolution of waves in the film is then calculated using Fresnel-Kirchhoff integration, where the diffracting structure serves as the aperture, and a twodimensional, attenuated Green's function describes the guided mode fields at the vacuum interface. This treatment of the Kirchhoff diffraction formula does not make approximations of the relative sizes of the aperture, wavelength, or distance to the screen, such as in Fresnel and Fraunhofer diffraction, and can be readily adapted to any type of propagating electromagnetic wave, independent of the underlying mechanism, as well as to arbitrarily shaped sources. We use the results from this analysis to compute the photoelectron emission rate, which derives from the complex sum of surface fields and incident light.

We experimentally study two photonic structures in an aberration-corrected PEEM using two-photon excitation and compare the electron micrographs to field-based calculations, where we find good agreement. As a result, we conclude that electromagnetic fields observed in PEEM are not significantly influenced by the photoelectrons. This may be an advantage compared to scanning probe techniques where the presence of the probe often affects the signal. We further submit that $n$ P-PEEM could be developed as a powerful visualization tool for the characterization of photonic and plasmonic structures where subwavelength sensitivity, nanometer precision, and detailed knowledge of near-field behavior are necessary. 


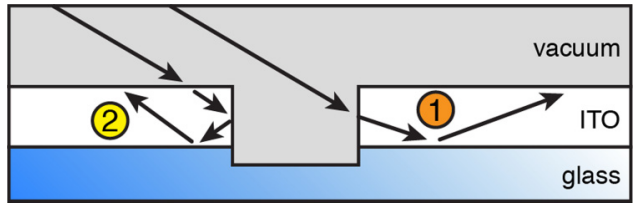

FIG. 1. (Color online) Ray paths of light incident on a single slit in a thin film dielectric waveguide. Path 1 is direct coupling, and path 2 is backscattered indirect coupling.

\section{THEORY}

The visualization of near-surface electromagnetic waves in PEEM relies on the interference between incident and scattered light at a specimen surface. To illustrate this point, consider a simple model (Fig. 1) of a single slit in a single-mode, thin-film dielectric waveguide. Light illuminates the entire thin film surface, which is otherwise uniform and planar. We can characterize the incident light with the scalar amplitude $E=$ $E_{0} e^{i \mathbf{k} \cdot \mathbf{r}}$ and wave vector $\boldsymbol{k}$, where $k=2 \pi / \lambda$ is the vacuum wavenumber and $\lambda$ is the vacuum wavelength of incident light. Some of the incident light scatters into the waveguide. A few nanometers from the step the guided wave can effectively be treated as a separate source $E^{\prime}=E_{1} e^{i \mathbf{k}^{\prime} \cdot \mathbf{r}}$, where $E_{1} / E_{0}$ is the coupling strength, $\boldsymbol{k}^{\prime}=N_{1} k \hat{\boldsymbol{e}}_{y}$ is the guided wave vector, and $N_{1}$ is the effective index [13].

The surface-projected wavenumbers of the incident and guided light are $k \sin \theta$ and $k N_{1}$, respectively, and the interference of the two waves at the surface is characterized by the wavenumber $k_{I}=k\left(N_{1}-\sin \theta\right)$, where $\theta$ is the angle of the incident light with respect to the surface normal. The interference maxima at the surface are separated by the distance $2 \pi / k_{I}$.

With $n$ photons required for the emission of a single electron, the rate of photoemission is proportional to the $n$th power of the total surface electromagnetic intensity, $R_{n} \propto$ $\left\|E_{\text {tot }}\right\|^{2 n}$, where $E_{\text {tot }}=E+E^{\prime}$ is evaluated at the surface. The $n \mathrm{P}$-PEEM image is a map of $R_{n}(\boldsymbol{r})$,

$$
R_{n} \propto\left\|E_{\text {tot }}\right\|^{2 n} \approx E_{0}^{2 n}+E_{0}^{2 n-1} E_{1} \cos \left(k_{I} r_{\perp}+\varphi\right),
$$

which shows the amplitude and phase of guided wave electromagnetic fields through the interference with the incident fields. Terms of order $E_{1}^{2}$ and higher are dropped, since $E_{0} \gg E_{1}$ in most cases, and $\varphi$ accounts for phase shifts between the two waves. The results of this example can be generalized to an arbitrarily shaped structure where there may be more than one $(j=1,2, \ldots)$ scattered wave $E_{j}^{\prime}$ directed in direction $\boldsymbol{k}_{j}^{\prime}$.

Coupling amplitude and polarization are calculated using a generalized transmission and reflection coefficient formalism, based on Fraunhofer diffraction and Maxwell's equations [14]. Light can be coupled into the waveguide through a directly illuminated face or through scattering at an indirectly illuminated face, as shown in Fig. 1, leading to anisotropy between forward and reverse-coupled light, with potentially different phase relationships with incident light. Unlike the single step example, we consider arbitrarily oriented faces, where fields have spatially varying transmission amplitude $A$. The complexity of the coupling calculation depends on the size and shape of the diffracting structure, as discussed in the Results section.

The wavenumbers of the guided modes supported in the dielectric thin film can be calculated from Maxwell's equations applied to an infinite slab model [15]. The waveguides considered here support two modes for each polarization, with wavenumbers $N_{j} k$, where the effective indices are $N_{1 \mathrm{TE}}, N_{1 \mathrm{TM}}$, $N_{2 \mathrm{TE}}$, and $N_{2 \mathrm{TM}}$. Due to the geometry of the milled grooves, incident transverse electric (TE)-polarized light can produce both TE- and transverse magnetic (TM)-polarized modes (and likewise for incident TM-polarized light); therefore, all four wavenumbers are potentially present. In practice, however, cross coupling between incident- and guided-mode polarization is at least an order of magnitude less than direct coupling. Thus, the calculation of the coupling amplitude $A$ can be reduced to a scalar computation by dropping crosscoupled modes without significant accuracy loss.

Propagation of the guided waves can then be computed from Huygens' principle, which treats every point of a scalar wavefront as a new source wavelet propagating outward in all directions. Since the guided waves are bound to the thin film layer, they can be represented by two-dimensional wavelets [16],

$$
G_{j}\left(\mathbf{r}, \mathbf{r}^{\prime}\right)=(i / 4) e^{-(\alpha / 2)\left|\mathbf{r}-\mathbf{r}^{\prime}\right|} H_{0}^{(1)}\left(N_{j} k\left|\mathbf{r}-\mathbf{r}^{\prime}\right|\right),
$$

where $j$ indicates the guided mode wavenumber, $\alpha$ is the absorption coefficient of the dielectric material, $\mathbf{r}$ is the sample location, $\mathbf{r}^{\prime}$ is the source location, and $H_{0}^{(1)}(x)$ is a zero-order Hankel function of the first kind. The complex field amplitude $E$ at any point $\mathbf{r}$ is the superposition of all the wavelets that originate at the boundary of the waveguide $\mathbf{r}^{\prime}$. Fresnel, and later Kirchhoff, Rayleigh, and Sommerfeld, developed a rigorous method to give $E(\mathbf{r})$ by integrating over the boundary value amplitude $E\left(\mathbf{r}^{\prime}\right)=A e^{i \mathbf{k} \cdot \mathbf{r}^{\prime}}$, where the boundary amplitude is the product of the incident wave $e^{i \mathbf{k} \cdot \mathbf{r}^{\prime}}$ and the complex coupling amplitude $A$.

With both the Green's function wavelet and the boundary amplitude, it is now possible to calculate the complex field amplitude $E_{j}(\mathbf{r})$ from the Fresnel-Kirchhoff integral [17],

$$
E_{j}(\mathbf{r})=\oint_{S}\left\{E\left(\mathbf{r}^{\prime}\right) \mathbf{n}^{\prime} \cdot \nabla^{\prime} G_{j}\left(\mathbf{r}, \mathbf{r}^{\prime}\right)-G_{j}\left(\mathbf{r}, \mathbf{r}^{\prime}\right) \mathbf{n}^{\prime} \cdot \nabla^{\prime} E\left(\mathbf{r}^{\prime}\right)\right\} d \ell^{\prime},
$$

where $S$ is a closed curve surrounding $\mathbf{r}, \mathbf{n}^{\prime}$ is the inwarddirected normal vector, and the integration is over the boundary $S$, which involves only the primed terms. Suitable approximations exist for partially unbounded regions such that many experimental situations can be computed. In our case four distinct electromagnetic field amplitudes, $E_{1 \mathrm{TE}}, E_{1 \mathrm{TM}}, E_{2 \mathrm{TE}}$, and $E_{2 \mathrm{TM}}$, correspond to each of the four guided waves, and separate calculations were carried out for each amplitude. The two-photon photoemission (2PPE) electron yield is finally computed from a superposition of these fields with the incident field amplitude,

$$
\begin{aligned}
R_{2} \propto & \| \hat{\mathbf{e}}_{\mathrm{TE}}\left(E_{0 \mathrm{TE}}+E_{1 \mathrm{TE}}+E_{2 \mathrm{TE}}\right) \\
& +\hat{\mathbf{e}}_{\mathrm{TM}}\left(E_{0 \mathrm{TM}}+E_{1 \mathrm{TM}}+E_{2 \mathrm{TM}}\right) \|^{4},
\end{aligned}
$$



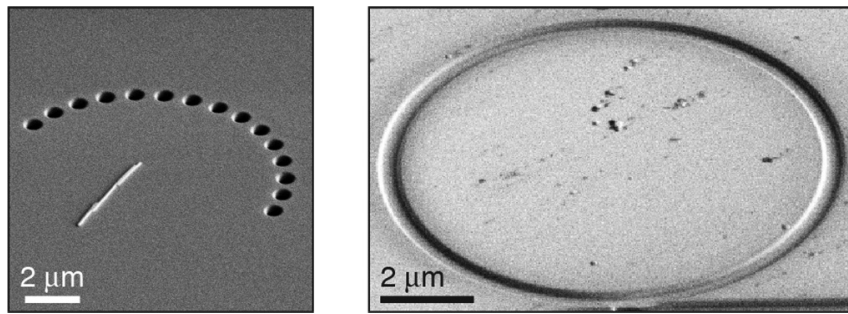

FIG. 2. Scanning electron micrographs of the two photonic structures in ITO thin films on glass. Images taken at an angle of $52^{\circ}$ with respect to the surface normal. (Left) First sample, a semicircle of holes with a gold nanowire at the center. (Right) Second sample, a circular disc defined by a 550-nm groove.

where $E_{0 \mathrm{TE}}$ and $E_{0 \mathrm{TM}}$ are the incident light components. As previously discussed, typically only one polarization is present in the calculation since cross-coupling is weak.

\section{EXPERIMENT}

Two photonic structures, an assembly of holes and a single dielectric disc, as shown in Fig. 2, were prepared by milling indium-tin-oxide (ITO) thin film on glass substrate with an FEI Strata 237 focused ion beam system. ITO is a transparent conductor, which in these experiments acts as a lossy dielectric waveguide. It was chosen because its electrical conductivity is sufficient to prevent local charging after electron emission. The assembly of holes consists of 15 holes with a diameter of $500 \mathrm{~nm}$ arranged in a 10- $\mu$ m-diameter semicircle. The second sample is a circular waveguide separated from the bulk film by a $550-\mathrm{nm}$-wide circular groove with a diameter of $15 \mu \mathrm{m}$.

At the incident vacuum wavelength of $410 \mathrm{~nm}$, ITO has an absorption coefficient of approximately $6 \times 10^{3} \mathrm{~cm}^{-1}$ and a refractive index of 2.14. The ITO film's thickness was $250 \pm$ $40 \mathrm{~nm}$, as determined by edge-view scanning electron microscopy (SEM). The substrate 0.2-mm-thick borosilicate glass has a refractive index of 1.53. Thus light can be confined to the ITO layer through total internal reflection.

These two photonic structures were chosen from an initial survey including variations on these patterns and several other shapes. In the samples selected, there are additional features that were not analyzed. Notably, the bottom portion of the first sample contains a gold nanowire that might support localized surface plasmon resonances (LSPRs), but does not contribute to the waveguide fields analyzed here.

An aberration-corrected PEEM $[10,18]$ was used to image photoelectrons excited by 410-nm laser pulses generated by a Spectra-Physics Mai Tai Ti:sapphire laser. The laser produces 80 -fs pulses at a wavelength of $820 \mathrm{~nm}$ and at repetition frequency of $80 \mathrm{MHz}$. The light pulses are upconverted to $410 \mathrm{~nm}$ with pulse energies of $2-\mathrm{nJ}$ and a $100-\mathrm{fs}$ duration using a Del Mar Photonics second harmonic generator. The upconverted pulses have a linewidth of $4 \mathrm{~nm}$, full width at half-maximum. The polarization direction of the laser was set with a tunable wave plate. The laser was incident at $60^{\circ}$ with respect to the surface normal and had a spot size of about $100 \mu \mathrm{m}$ in diameter. We found that the 2PPE yield obtained with TM polarized light was about six times greater than the yield obtained with TE light, which we mostly attribute to the vectorial nature of the photoelectric effect [19].

\section{RESULTS}

Figures 3 and 4 show PEEM micrographs of the semicircular hole assembly and groove encircling a disc-shaped waveguide region, respectively. The one-photon photoemission (1PPE) images show topographic features, similar to an SEM image. Modulations in electron emission due to surface light interference are less pronounced. On the other hand, the 2PPE images show much stronger contrast resulting from the superposition of guided fields from curved sources and the incident light. Guided waves refracted into the disc structure in Fig. 3 converge at multiple foci, with the dominant focus attributable to the forward direction and a weaker, secondary focus from the reverse direction found just below the primary focus. Bright line patterns diverge from the structure in Fig. 4 as a result of constructive interference between two or more holes. The presence of multiple waveguide modes leads to the fractitious appearance of beating in Fig. 3 and the line patterns in Fig. 4.

Analysis of Figs. 3 and 4 yields the guided mode effective indices and relative mode amplitudes, which we can quantitatively compare to theoretical calculations to complement qualitative comparison of the experimental and theoretical images. We begin with the fields of a single, representative
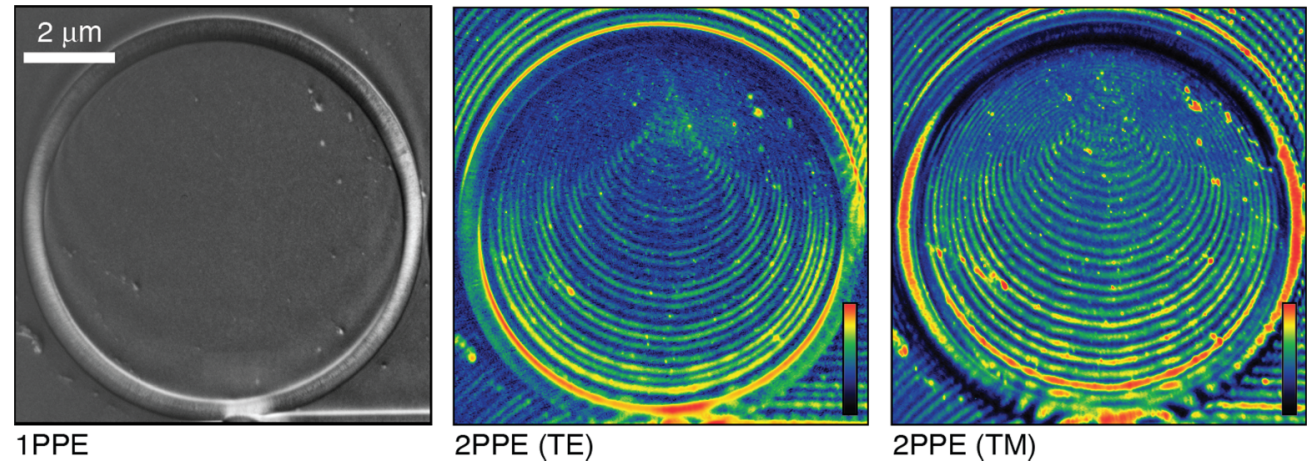

FIG. 3. (Color online) PEEM micrographs of a waveguide bounded by a circular groove. Light is incident from the bottom edge of the images. The 1PPE image shows the milled groove down to the glass substrate as a lighter shade. The 2PPE images (in false color) show modulations in the surface electromagnetic field due to interference between incident and guided light. 


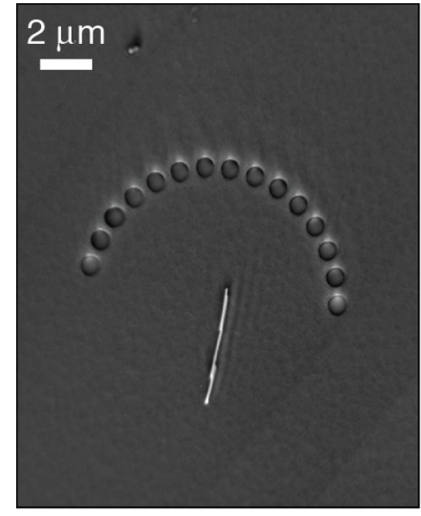

1PPE

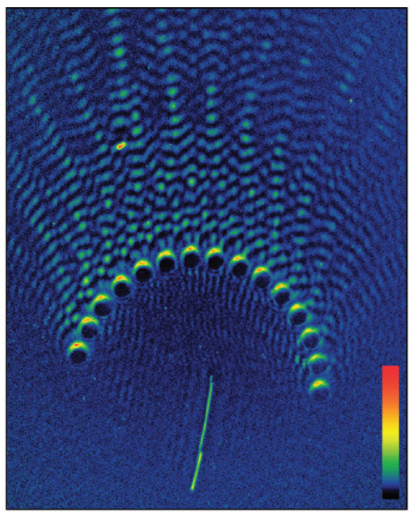

2PPE (TE)

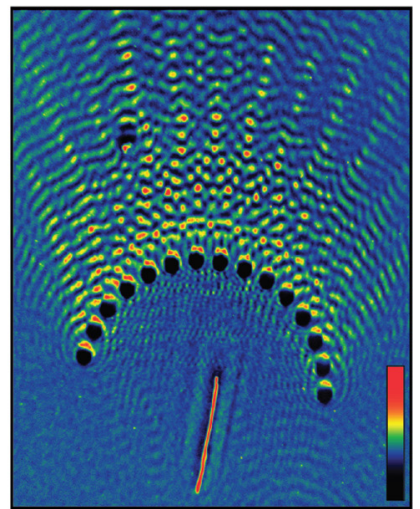

2PPE (TM)

FIG. 4. (Color online) PEEM micrographs of a semicircle of holes. Light is incident from the bottom edge of the images. The 1PPE image shows topographic features, similar to an SEM image, as well as some diffraction. The 2PPE images (in false color) show modulations in the surface electromagnetic field due to interference between incident and guided light.

hole, shown in Fig. 5, which is reconstructed from an average of the fields surrounding a selection of holes in the assembly. Figure 6 shows line profiles bisecting the hole in the direction of incident light. The effective indices and relative strengths of the two guided modes can be more readily determined from the Fourier transform power spectra of the line profiles, also shown in Fig. 6. Adding the in-plane component of the incident wavenumber to the interference wavenumber gives the guided mode wavenumber, or, in terms of the effective index, $N=$ $k_{l} / k_{0}+\sin \theta$. Thus, $N_{1 \mathrm{TE}}=1.78, N_{2 \mathrm{TE}}=2.05, N_{1 \mathrm{TM}}=1.68$, and $N_{2 \mathrm{TM}}=2.02$. Using these effective indices, we calculate an ITO film thickness of $265 \mathrm{~nm}$, which is consistent with the $250 \pm 40 \mathrm{~nm}$ measured in SEM [15]. The relative amplitudes can also be determined from the peak intensities of the Fourier transform power spectrum, giving 0.28 (TE) and 0.38 (TM). These values conform to within $6 \%$ with a Fraunhofer diffraction model based on the Airy disc, $P_{2} / P_{1}=E_{2} / E_{1} \approx$ $\left[J_{1}\left(\zeta_{2}\right) \zeta_{2}^{-1}\right] /\left[J_{1}\left(\zeta_{1}\right) \zeta_{1}^{-1}\right]$, where $J_{1}(x)$ is a Bessel function of the first kind, and $\zeta_{1}=k_{0} d \sin \left(\theta-\sin ^{1} N_{j} / n_{\text {ITO }}\right)$. Figure 3 gives similar effective indices as the holes since the film is roughly the same thickness. Guided mode relative amplitudes in the disc, $\left(E_{2} / E_{1}\right)_{\mathrm{TE}}=0.84$ and $\left(E_{2} / E_{1}\right)_{\mathrm{TE}}=0.41$, are different from hole diffraction, conforming instead to a slit diffraction model, where $\sin \zeta_{j}$ replaces $J_{1}\left(\zeta_{j}\right)$, to within $6 \%$.

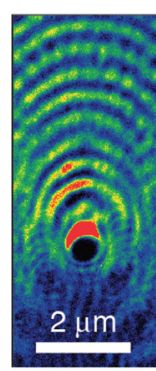

TE (exp.)

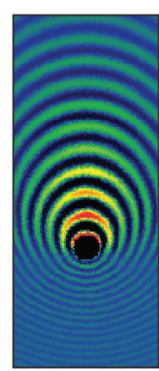

TE (sim.)

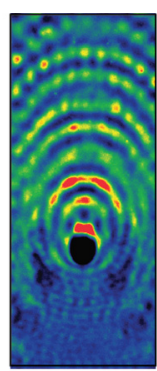

TM (exp.)

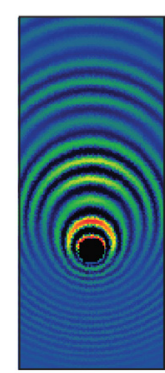

TM (sim.)
FIG. 5. (Color online) Experimental and simulated 2PPE interference patterns generated by a single hole. The experimentally derived patterns are composite sums of the 2PPE near each hole in Fig. 4.
Next, we compute the expected photoelectron yield intensity of our theory. We begin by calculating the coupling amplitude, $A_{j}$. In both cases, we use simple approximate expressions to expedite numerical integration of the Kirchhoff formula. We found that fields diffracted through a hole could be accurately modeled with a real transmission coefficient that is unity for the top edge and 0.66 for TE and 1.0 for TM modes for the bottom edge of a hole. Because of the extended nature of the circular groove, it was necessary to use amplitudes that vary more sensitively with position around the circular groove edge. These were calculated using Fresnel transmission coefficients expressions with variable incident angle [14]. The results for this calculation are shown in Fig. 7. Next, we used the calculated guided mode wave numbers, as given above, in order to obtain a scalar field distribution for each guided mode. Individual field distributions $E_{j}$ are calculated from a numerical integration of the Kirchhoff formula. Subsequently the total surface fields are linear superpositions of the incident wave field and the two guided mode fields weighted according to their diffractive amplitudes, given by the Bessel or sinc
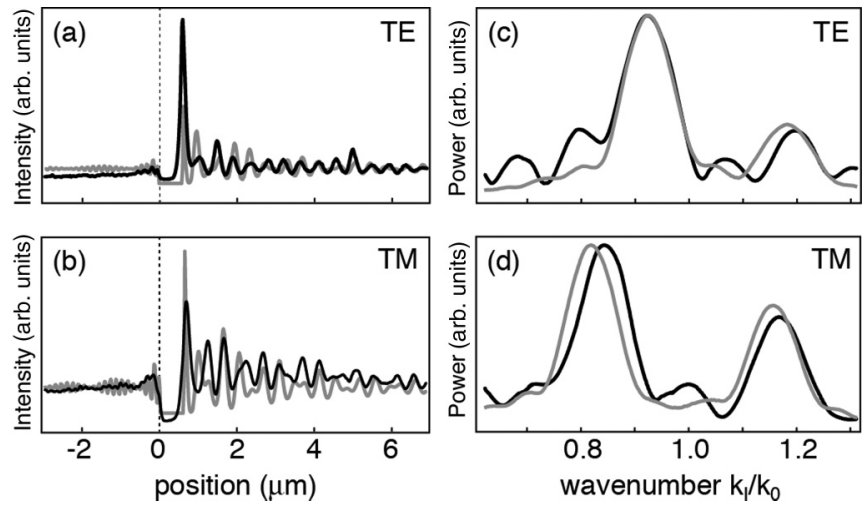

FIG. 6. 2PPE pattern from a single $0.5-\mu \mathrm{m}$ hole. (a), (b) Line profiles taken in the direction of incident light, with simulated line profile shown in light gray. (c), (d) Fourier transform power spectra of the line profiles plotted vs interference wavenumbers normalized by vacuum wavenumber. Simulated spectra are shown in light gray, experimental data in heavy black. 

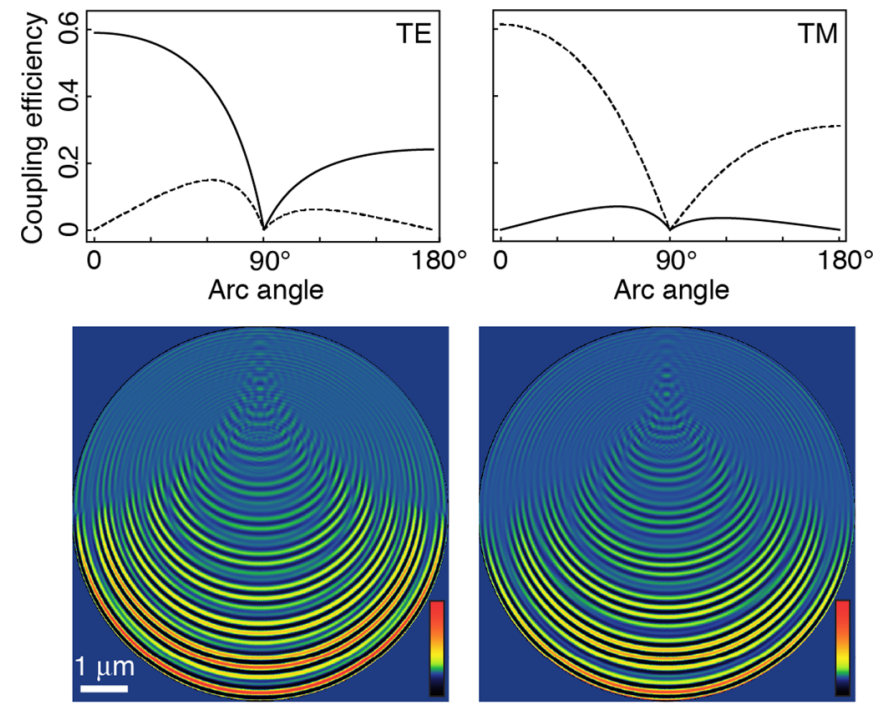

TE

TM

FIG. 7. (Color online) (Top) Calculated efficiency of TE and TM incident light for generating TE-like (solid line) and TM-like (dotted line) guided modes in the ITO, with $0^{\circ}$ position at the bottom and $180^{\circ}$ at the top of the disc. (Bottom) Simulated 2PPE pattern for the disc structures.

functions above. Constructing the field distribution of multiple holes requires a further superposition of individual hole fields. The relative 2PPE intensities are then computed from the total surface fields using Eq. (1). Figure 5 shows the calculated photoelectron yield intensities of a single hole, with quantitative comparisons to experimental data given in Fig. 6. Theoretical PE yields of the more advanced structures of a disc and semicircle of holes are shown in Figs. 7 and 8.

Overall our calculations in Figs. 6, 7, and 8 show excellent agreement with the experimental 2PPE electron micrographs in Figs. 3 and 4. Single hole simulations reproduce the observed asymmetric interference patterns, with bright, widely spaced interference maxima in the direction of the incident light wave vector, weak, finely spaced maxima in the opposite direction, and a smooth gradient of maxima intensity and spacing along the sides. Both modeled and experimental images of the hole assembly have similar patterns of speckles, diverging lines of constructive interference maxima in the forward direction and finer lines of interference maxima

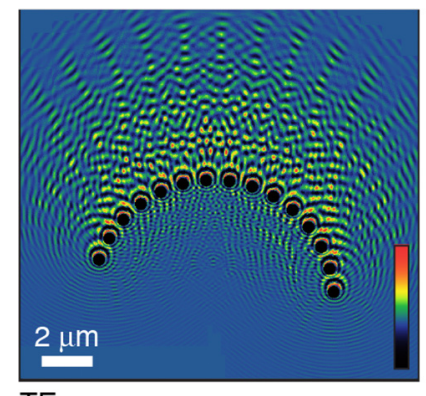

TE

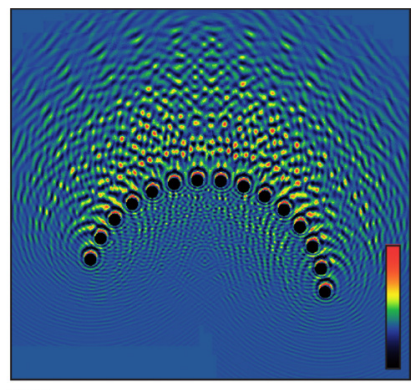

TM
FIG. 8. (Color online) Simulated 2PPE patterns constructed from a superposition of single hole simulations. Individual hole line profiles and spectra are compared to experiment in Fig. 6. parallel to the incident wave vector in the reverse direction. In the disc structure, the model maxima follow the contour of the groove edge as observed. Additionally, the locations and intensities of foci as well as the beating between the two guided modes are accurately reproduced. Differences between model and observed 2PPE images highlight the depth of information in present 2P-PEEM electron micrographs. These are especially apparent in near-field zones, around groove and hole edges, and are thus likely the result of more complicated optical and geometric properties than in the idealized simulation.

\section{DISCUSSION AND CONCLUSIONS}

The electron micrographs presented in Figs. 3 and 4 exhibit nanometer-scale resolution. In the direction opposite to the incident wave vector the interference maxima are separated by $2 \pi / k_{I}=\lambda /(N+\sin \theta) \approx 130 \mathrm{~nm}$. Features near grooveand hole-edges exhibit even higher resolution, typically about $50 \mathrm{~nm}$. Thus, $n$ PPE electron micrographs provide subdiffraction visualizations of electromagnetic field distributions at the surface of materials that are accurate and complete pictures of light dynamics. The resolution of these images is not limited by the wavelength of illumination. Instead, in the current work, low electron luminosity is the primary resolution-limiting factor. With the appropriate sample and lighting conditions the resolution might approach $5 \mathrm{~nm}$, similar to ultraviolet aberration-corrected PEEM [10]. Since longer exposure times may result in stability issues, higher laser pulse rates may be the best way to improve the luminosity and thereby resolution $[20,21]$.

The observation of guided waves complements observations of other optical phenomena [1-5,7-10], indicating that $n \mathrm{P}-\mathrm{PEEM}$ can observe a wide range of optical processes. All propagating surface electromagnetic fields, such as surface plasmon polaritons and surface photonic waves, can be quantitatively analyzed using the same techniques presented here and in similar analyses [4-6,9], as we have recently suggested [7]. This analysis can be used to measure wave speed, propagation length, and relative amplitudes, differentiating between the various forms of surface electromagnetic phenomena and leading to a better understanding of the physics of nanophotonic structures. To complement the earlier analysis, we now demonstrate a more general method for calculating surface fields and relative photoemission rates of more complicated optical structures based on the scalar wave diffraction theory of Kirchhoff.

Thus, $n$ P-PEEM combines the ability to observe a wide range of electromagnetic phenomena with subwavelength sensitivity and excellent resolution. We believe that $n$ P-PEEM could ultimately visualize even weak optical processes with a resolution of $\lambda / 50$. Such intimate knowledge would open the near-field range to quantitative experimental analysis and would allow new approaches for the development of photonic and plasmonic metamaterials.

\section{ACKNOWLEDGMENT}

This work was supported by the US DOE Basic Science Office Contract No. DE-FG02-07ER46406. 
[1] A. Kubo, K. Onda, H. Petek, Z. Sun, Y. S. Jung, and H. K. Kim, Nano Lett. 5, 1123 (2005).

[2] L. Douillard, F. Charra, Z. Korczak, R. Bachelot, S. Kostcheev, G. Lerondel, P.-M. Adam, and P. Royer, Nano Lett. 8, 935 (2008).

[3] P. Melchior, D. Bayer, C. Schneider, A. Fischer, M. Rohmer, W. Pfeiffer, and M. Aeschlimann, Phys. Rev. B 83, 235407 (2011).

[4] L. Zhang, A. Kubo, L. Wang, H. Petek, and T. Seideman, Phys. Rev. B 84, 245442 (2011).

[5] C. Lemke, T. Leißner, S. Jauernik, A. Klick, J. Fiutowski, J. Kjelstrup-Hansen, H.-G. Rubahn, and M. Bauer, Opt. Express 20, 12877 (2012).

[6] J. P. S. Fitzgerald, R. C. Word, S. D. Saliba, and R. Könenkamp, Phys. Rev. B 87, 205419 (2013).

[7] R. C. Word, J. P. S. Fitzgerald, and R. Könenkamp, Appl. Phys. Lett. 103, 021118 (2013).

[8] C. Lemke, C. Schneider, T. Leißner, D. Bayer, J. W. Radke, A. Fischer, P. Melchior, A. B. Evlyukhin, B. N. Chichkov, C. Reinhardt, M. Bauer, and M. Aeschlimann, Nano Lett. 13, 1053 (2013).

[9] R. C. Word, J. P. S. Fitzgerald, and R. Könenkamp, Opt. Express, 21, 30507 (2013).
[10] R. Könenkamp, R. C. Word, G. F. Rempfer, T. Dixon, L. Almaraz, and T. Jones, Ultramicroscopy 110, 899 (2010).

[11] E. Bauer, M. Mundschau, W. Swiech, and W. Telieps, Ultramicroscopy 31, 49 (1989).

[12] G. F. Rempfer and O. H. Griffith, Ultramicroscopy 47, 35 (1992).

[13] L. I. Chelaru, M. Horn-von Hoegen, D. Thien, and F. J. Meyer zu Heringdorf, Phys. Rev. B 73, 115416 (2006).

[14] J. D. Jackson, Classical Electrodynamics, 3rd ed. (Wiley, New York, 1998), p. 479.

[15] A. Yariv, Optical Electronics (Saunders College Publishing, Philadelphia, 1991).

[16] W. Singer, M. Totzeck, and H. Gross, Handbook of Optical Systems, Physical Image Formation (Wiley, Weinheim, Germany, 2006).

[17] J. D. Jackson, Classical Electrodynamics, 3rd ed. (Wiley, New York, 1998), p. 304.

[18] G. F. Rempfer, J. Appl. Phys. 67, 6027 (1990).

[19] R. M. Broudy, Phys. Rev. B 3, 3641 (1971).

[20] S. M. Schramm, A. B. Pang, M. S. Altman, and R. M. Tromp, Ultramicroscopy 115, 88 (2012).

[21] A. Bartels, D. Heinecke, and S. A. Diddams, Opt. Lett. 33, 1905 (2008). 